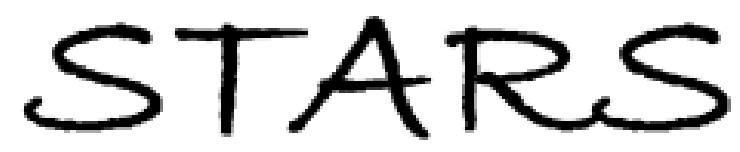

University of Central Florida

STARS

Faculty Bibliography 1990s

Faculty Bibliography

$1-1-1993$

\title{
K-Shell Auger Spectrum Of Atomic Oxygen
}

C. D. Caldwell

University of Central Florida

M. O. Krause

Find similar works at: https://stars.library.ucf.edu/facultybib1990

University of Central Florida Libraries http://library.ucf.edu

This Note is brought to you for free and open access by the Faculty Bibliography at STARS. It has been accepted for inclusion in Faculty Bibliography 1990s by an authorized administrator of STARS. For more information, please contactSTARS@ucf.edu.

\section{Recommended Citation}

Caldwell, C. D. and Krause, M. O., "K-Shell Auger Spectrum Of Atomic Oxygen" (1993). Faculty Bibliography 1990s. 654.

https://stars.library.ucf.edu/facultybib1990/654

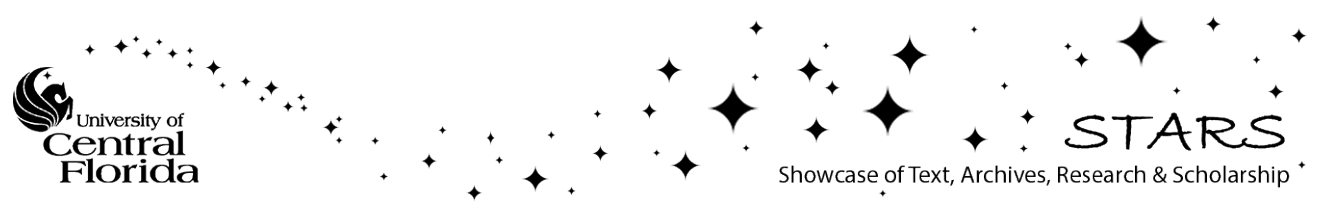




\title{
$K$-shell Auger spectrum of atomic oxygen
}

\author{
C. D. Caldwell \\ Department of Physics, University of Central Florida, Orlando, Florida 32816-2385 \\ M. O. Krause \\ Oak Ridge National Laboratory, Oak Ridge, Tennessee 37831-6201
}

(Received 4 December 1992)

\begin{abstract}
We have observed the $K$-shell Auger spectrum in atomic oxygen. A total of ten transitions originating from the decay of the ${ }^{4} P$ and ${ }^{2} P$ states of the $\mathrm{O}^{+}\left(1 s^{-1}\right)$ ion are identified and assigned to final states of the $\mathrm{O}^{2+}$ ion. Relative intensities for each of these transitions are obtained, allowing the extraction of the dependence of the Auger decay on the multiplet structure in both the initial and final states of this basic open-shell atom.
\end{abstract}

PACS number(s): 32.80.Hd, 32.30.- r

Oxygen, a simple omnipresent species, plays a vital role in chemical reactions, surface physics, and processes in the upper atmosphere. Yet a complete understanding of the detailed electronic behavior of oxygen is still lacking. This applies, in particular, to inner-shell ionization and its consequences. A good understanding of atomic oxygen, in addition to molecular oxygen, is critical for the interpretation of high-energy absorption data and for isolation of those surface features that might be essentially atomic in origin. At the same time, the study of innershell absorption in atomic oxygen opens new paths for benchmark measurements on Auger processes in the first-row elements, particularly as they pertain to openshell atoms $[1,2]$. Although most first-row elements are basic building blocks of matter, both animate and inanimate, the phenomenon of the Auger effect has been addressed experimentally only for beryllium [3] and for boron and carbon [4], or for their isoelectronic ions [5], but not for nitrogen, oxygen, and fluorine.

In atomic oxygen the $K-L L$ Auger spectrum can be studied as a function of the multiplet terms occurring in both the initial and final states. None of the few theoretical studies made around $Z=8$ goes beyond the independent-particle model [6,7], although it had been shown for neon, after many years of endeavor, that electron correlation is of crucial importance in the interpretation of the Auger spectrum of this closed-shell atom [1,2]. We present in this Rapid Communication a detailed measurement and interpretation of the $K$-shell Auger spectrum of atomic oxygen. We isolate the spectrum with regard to the production of the various multiplets of the $\mathrm{O}^{+}\left(1 s^{-1}\right)$ ion as well as to the influence of the multiplet structure of the $\mathrm{O}^{2+}$ ion on the intensities.

The experiment was performed at the University of Wisconsin Synchrotron Radiation Center on a 10-m toroidal-grating monochromator. The oxygen atoms were produced in a microwave discharge tube designed to serve as a source for the electron spectrometer, using molecular oxygen as the precursor. Details of both the design and operation of the discharge tube and the electron spectrometer have been given in earlier work $[8,9]$. In order to enhance the atomic yield we added a small quantity of $\mathrm{N}_{2}$ to the $\mathrm{O}_{2}$. All measurements were performed at a photon energy of $590 \mathrm{eV}$, well above the oxygen $K$ edge, and the Auger spectra were recorded with a resolution of $0.5 \mathrm{eV}$ full width at half maximum. Each measurement was performed with the discharge on and with the discharge off to generate a molecular spectrum that could be subtracted from the total to reveal the atomic features.

In Fig. 1 are given examples of (a) the spectrum with
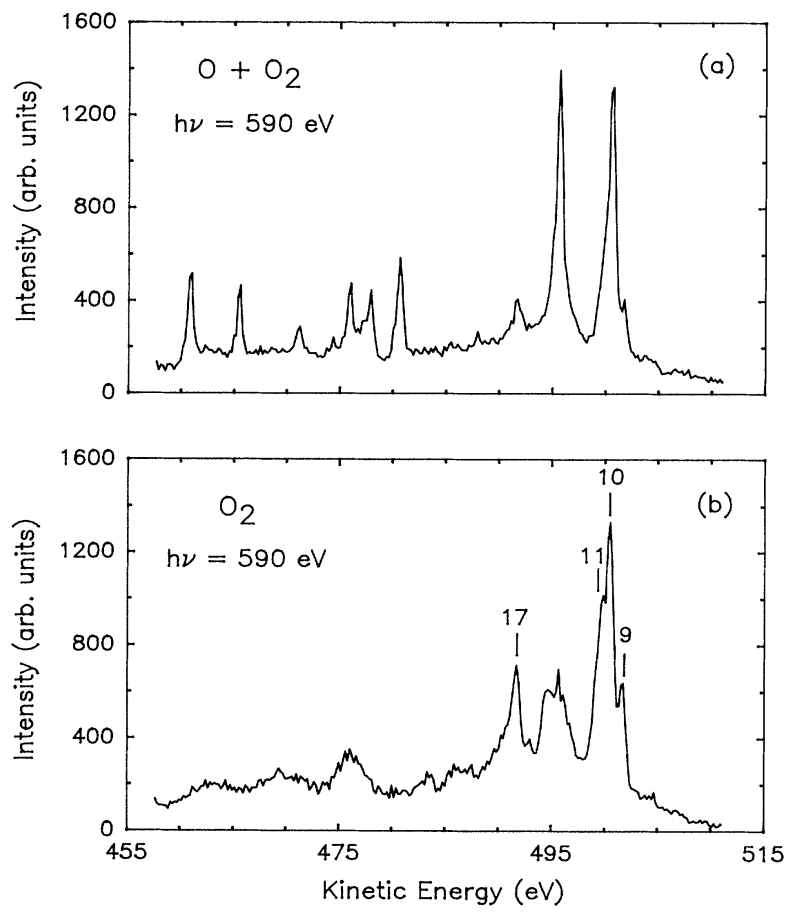

FIG. 1. Auger spectra of (a) atomic plus molecular oxygen and (b) molecular oxygen taken at a photon energy of $590 \mathrm{eV}$. The labels in the molecular spectrum correspond to those given by Larsson et al. (Ref. [13]). Feature 10 at $500.5 \mathrm{eV}$ is that chosen for the normalization of the energy scale. These curves are raw data, with no intensity normalization with respect to each other. Scans to energies up to $525 \mathrm{eV}$ and down to $450 \mathrm{eV}$ reveal no additional structure. 
the discharge on, including both atomic and molecular contributions, and (b) the Auger spectrum of $\mathrm{O}_{2}$ alone, taken with the discharge off. A characteristic of these spectra is the close overlap between the Auger transitions in the atom and the Auger transitions in the molecule. This is particularly true for the strongest features of both spectra. However, many of the weaker atomic transitions appear almost free of interfering molecular contributions.

Figure 2 depicts the atomic spectrum that results when two spectra of the type given in Fig. 1 are subtracted. In each measurement simultaneous spectra were recorded in three independent analyzers, oriented at $90^{\circ}$ with respect to each other. As we discerned no dependence of the relative intensity distribution of the spectra on the angle of observation, the spectrum shown in Fig. 2 is a direct addition of three of these simultaneous measurements. We are able to clearly identify ten features that can be assigned to the $K$-shell Auger spectrum of atomic oxygen. Also shown in Fig. 2 is a fit of the spectrum to a combination of Pearson-7 functions [10] representing the features. Line positions and relative intensities are obtained from the centers and areas under the fitted peaks, respectively. Relative energy positions for each of the transitions are given in Table I, using the position of the most intense peak in the atomic spectrum as ap apropriate "zero" for the energy scale. The errors are derived as the standard deviation of a series of six independent determinations. Also given in Table I are relative energy positions of the corresponding hole states in the $\mathrm{O}^{2+}$ ion that form final states for the Auger transitions, taken from the tabulation by Moore [11]. As can be seen, the separation between the various lines corresponds quite well with the spectroscopic values [11].

While the tabulation by Moore allows identification of the possible final states of $\mathrm{O}^{2+}$, there exist no data on the energy positions of the initial states corresponding to the $1 s$ hole. Larkins [12] has performed a Hartree-Fock calculation of the energies of the four multiplets and obtains

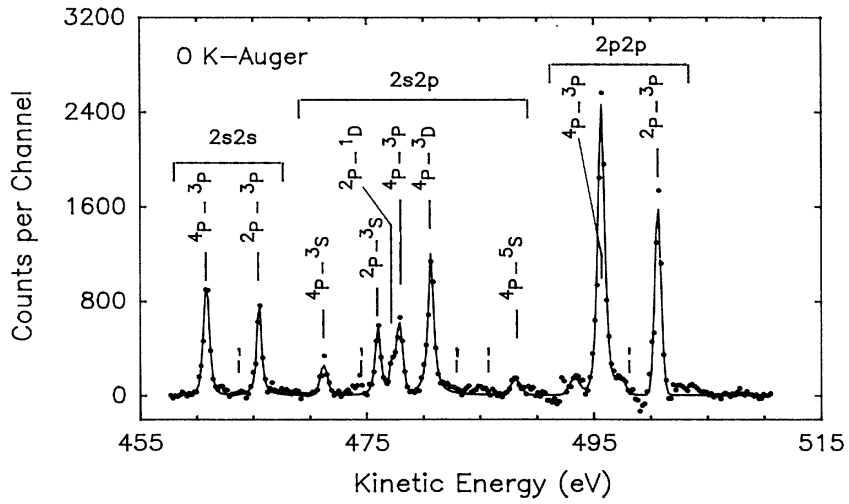

FIG. 2. $K$-shell Auger spectrum of atomic oxygen. Points represent the data; the solid line represents the fit of the spectrum to a group of Pearson-7 functions, with the peak height and width variable for each feature. Two dummy peaks were introduced near $495 \mathrm{eV}$ to account for residual signal. No transmission correction has been applied. Calculated positions of ${ }^{2} \boldsymbol{P}$ transitions too weak to be ascertained are given by vertical dashed lines.
TABLE I. Energies and relative decay strengths of the components of the Auger spectrum of atomic oxygen.

\begin{tabular}{|c|c|c|c|}
\hline \multirow[t]{2}{*}{ Transition } & \multicolumn{2}{|c|}{$\Delta E(\mathrm{eV})$} & \multirow{2}{*}{$\begin{array}{l}\text { Relative } \\
\text { intensity }\end{array}$} \\
\hline & This work & Moore $^{\mathrm{a}}$ & \\
\hline $1 s^{2} P \rightarrow 2 p 2 p{ }^{3} P$ & $+4.87(8)$ & $+4.87^{\mathrm{b}}$ & $1.4(2)$ \\
\hline $1 s^{4} P \rightarrow 2 p 2 p{ }^{3} P$ & $0.0^{\mathrm{c}}$ & 0.0 & $3.0(7)$ \\
\hline $1 s^{4} P \rightarrow 2 s 2 p{ }^{5} S$ & $-7.13(40)$ & -7.452 & $0.18(6)$ \\
\hline $1 s^{4} P \rightarrow 2 s 2 p^{3} D$ & $-14.86(5)$ & -14.857 & 1.00 \\
\hline $1 s^{4} P \rightarrow 2 s 2 p{ }^{3} P$ & $-17.62(13)$ & -17.627 & $0.44(4)$ \\
\hline $1 s^{2} P \rightarrow 2 s 2 p{ }^{1} D$ & $-18.42(18)$ & $-18.295^{b}$ & $0.16(3)$ \\
\hline $1 s^{2} P \rightarrow 2 s 2 p^{3} S$ & $-19.59(12)$ & $-19.540^{b}$ & $0.58(5)$ \\
\hline $1 s^{4} P \rightarrow 2 s 2 p^{3} S$ & $-24.39(7)$ & -24.410 & $0.20(2)$ \\
\hline $1 s^{2} P \rightarrow 2 s 2 s^{3} P$ & $-30.24(3)$ & $-30.229^{b}$ & $0.50(5)$ \\
\hline $1 s^{4} \mathrm{P} \rightarrow 2 s 2 s^{3} \mathrm{P}$ & $-35.04(8)$ & -35.169 & $0.64(1)$ \\
\hline
\end{tabular}

${ }^{a}$ Reference [11]. All triplet energies are based on weighted averages of the energies of the three fine-structure components.

${ }^{b}$ Position derived from the energy level of the final state as given by Moore with experimental splitting of $4.87 \mathrm{eV}$ added.

${ }^{c}$ The absolute energy for this transition is $495.7(3) \mathrm{eV}$. (See text.)

the following spacings between the ${ }^{4} P$ final state and the other three: ${ }^{2} P,-4.67 \mathrm{eV} ;{ }^{2} D,-4.40 \mathrm{eV} ;{ }^{2} S,-8.56 \mathrm{eV}$. As a corollary to the Auger spectrum, we measured the photoelectron spectrum (PES) originating from $1 s$ ionization. The $\mathbf{O}(1 s)$ PE spectrum is displayed in Fig. 3, following subtraction of the molecular contribution. The fact that only two peaks are visible in the spectrum indicates that, based on the spacings given by Larkins, we are not producing all of the possible final states. In our discharge we expect to find only the ${ }^{3} P$ ground state of oxygen, as the first excited state lies $1.97 \mathrm{eV}$ higher in energy. Larkins gives the ${ }^{4} P$ state as the ground state of the configuration $1 s 2 s^{2} 2 p^{4}$. If we make the assumption that the ionization predominantly gives rise to the ${ }^{4} P$ final state, which is consistent with the PES analysis and with

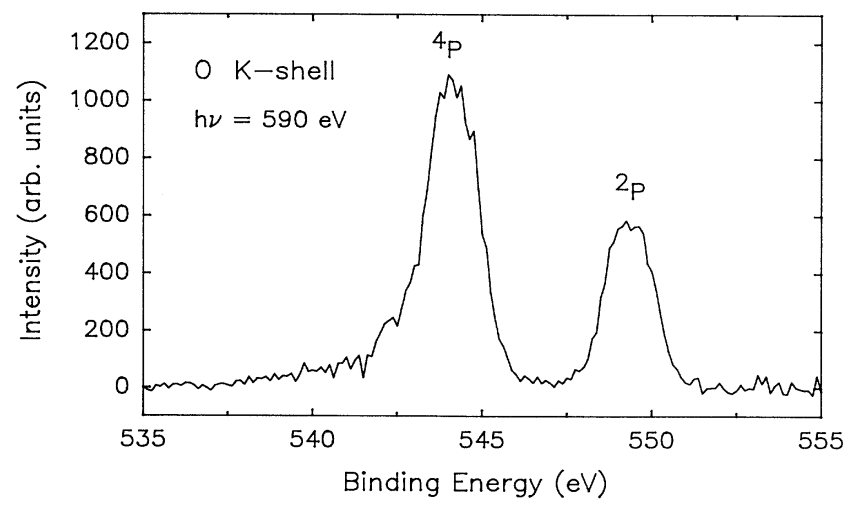

FIG. 3. Photoelectron spectrum of $K$-shell ionization of atomic oxygen recorded at an angle of $57^{\circ}$. The two prominent features are assigned to the ${ }^{4} P$ and ${ }^{2} P$ initial states produced by the coupling of the $1 s$ hole with the $2 p^{4}$ configuration. The residual intensity on the left foot of the ${ }^{4} P$ feature is attributed to a contaminant of either $\mathrm{CO}_{2}$ or $\mathrm{N}_{2} \mathrm{O}$ produced by the discharge. 
ionization from the ${ }^{3} P$ ground state, we can separate the ten Auger transitions into two series. The first originates from the ${ }^{4} P$ state of $\mathrm{O}^{+}$and gives rise to the strongest feature in Fig. 2. Once we establish this as a starting point, we can identify all the allowed transitions originating from the ${ }^{4} P$ initial state. (See Table I.) The remaining Auger transitions and the second feature in the PE spectrum must then arise from the production of the ${ }^{2} P$ or ${ }^{2} D$ state. Unfortunately, the PES resolution of the experiment, $1.5 \mathrm{eV}$ at best, did not permit the separation of these levels. We have chosen to assign the remaining Auger lines to the ${ }^{2} P$ series for three reasons. The first is the closer overlap between the calculated energy separation [12], $4.67 \mathrm{eV}$, and that measured in the PES experiment, 4.97(12) eV, and by means of the Auger transitions, 4.83(7) eV. The second is the absence of an Auger transition at $456.4 \mathrm{eV}$, which would only be allowed if originating from the ${ }^{2} D$ initial state [12]. Finally, all the Auger lines have essentially the same widths. If the ${ }^{2} P$ and ${ }^{2} D$ states were both involved equally in the transitions, we would expect an increase in width by approximately 0.2 $\mathrm{eV}$ due to the separation [12] of $0.27 \mathrm{eV}$.

A calibration of the energy scale with the known value of the main $K$-shell Auger feature of the molecular spectrum allows the extraction of energies of the Auger transitions. We use the energy of the molecular Auger transition [13,14], designated as No. 10 by Larsson et al. [13], $500.50 \mathrm{eV}$, to arrive at an energy of $E=500.6(2) \mathrm{eV}$ for the $1 s^{2} P \rightarrow 2 p 2 p{ }^{3} P$ Auger line. Similarly, we used the molecular $K$-level energy of $543.7(1) \mathrm{eV}$ as the weighted average of the spin-orbit pair (Ref. [13]) to obtain $E=544.3$ (3) for the $\mathrm{O} 1 s^{4} P$ level energy. This value agrees within $0.2(3) \mathrm{eV}$ with that derived from our corresponding Auger energy, 495.7(3) eV, and the spectroscopic energy of $48.80 \mathrm{eV}$ for the $J$-averaged ground state of $\mathrm{O}^{2+}$ (Ref. [11]). The shift of the average $K$-shell binding energy is found to be $-2.4(3) \mathrm{eV}$ when going from the $\mathrm{O}$ atom to the $\mathrm{O}_{2}$ molecule.

The determination of the intensities of the various transitions is complicated by the fact that the electrons were severely retarded in kinetic energy in order to obtain a suitable resolution in the experiment. Retardation in the electron spectrometer is accomplished by applying a voltage to the cell defining the source region. In our case this amounted to as much as $450 \mathrm{~V}$ for the highenergy Auger electrons. We recorded the Auger spectrum in two different modes of operation to minimize uncertainties in the intensities. In the first a staircase voltage is applied to the plates of the electron analyzer, thus altering the selected kinetic energy while maintaining constant retardation (plate scan). In the second the pass energy of the electron analyzers is held fixed while a staircase voltage is applied to the source cell (source scan). The response of the electron spectrometer in each of these scan modes was calibrated through measurements of the photoelectron spectrum of neon at different retardation voltages and photon energies and, in addition, by comparison with existing data for the $\sigma(2 s): \sigma(2 p)$ ratio [15]. In both cases we determined that, at these severe retardations, there exists a deviation from the ideal transmission function of the analyzers across the region of the scan. In the case of the plate scan, the deviation from $I \propto 1 / E_{\text {kin }}$ is quite pronounced, but in the case of the source scan, the deviation from $I=$ const is rather mild. Thus, the intensity ratios that we give in Table I are a weighted average of the corrected intensities taken using both scan modes, with more weight being given to those obtained using the source scan.

The results for the intensities of each of the ten observed lines are listed in Table I. The errors given reflect the uncertainties introduced by the retardation correction as well as inaccuracies introduced by the subtraction. The results are presented as the observed intensities relative to the isolated $1 s{ }^{4} \mathrm{P} \rightarrow 2 s 2 p{ }^{3} \mathrm{D}$ transition; they do not include any correction due to the different populations of the initial $1 s$ hole state. From an analysis of seven measurements in the PES mode we determine a value of 0.53 (3) for the ratio $I\left({ }^{2} P\right): I\left({ }^{4} P\right)$. This is consistent with a statistical distribution into these two final states and lends credence to the assignment of the Auger spectra as arising from the two $P$ states.

It is of interest to compare the group intensities arising from one of the initial states with related data. The branching ratios observed for the $1 s^{4} P$ series, $\alpha(2 p 2 p): \alpha(2 s 2 p): \alpha(2 s 2 s)=0.55: 0.33: 0.12$, agree reasonably well with the branching ratios of 0.43:0.41:0.16 calculated for O-like neon [6], but poorly with the experimental ratios of 0.71:0.23:0.06 for $\mathrm{Ne}$ [16], even if a correction were applied for the smaller number of $2 p$ electrons present in oxygen. Chen, Larkins, and Crasemann [7] give ratios of 0.58:0.37:0.06 for the $O$ atom. The difference between single-particle theory and experiment indicates that the $2 s 2 s{ }^{3} P$ transition borrows intensity from $2 p 2 p^{3} P$ and $2 s 2{ }^{3} P$. This final-ionic-state interaction (FISI) appears even stronger in the $1 s^{2} P$ series, in which the $2 s 2 p{ }^{3} P$ line is not observed but the $2 s 2 s^{3} P$ line is further enhanced. Pronounced FISI is not unexpected, as it is known from neon that the $2 p 2 p{ }^{1} S$ and $2 s 2 s{ }^{1} S$ states interact strongly. Bhalla's results [6] might indicate that the $2 s$ orbital contracts strongly with increasing nuclear charge, a feature reminiscent of our observation in beryllium [17].

The results reported in this Rapid Communication represent only the beginning of Auger studies on this fundamental atomic species. Experiments are currently underway to isolate the atomic excitations of the type $1 s \rightarrow n p$ and observe their radiationless decay. Theoretical single-particle models, seen to be as inadequate for oxygen as they once were for neon, need to be extended to include final-ionic-state interactions and finalcontinuum-state interactions. Special attention might have to be paid to possible mixing of the initial ionic states created in the photoionization of this open-shell atom. This question may be intertwined with the weakness, or absence, of the allowed ${ }^{2} D$ and ${ }^{2} S$ ionic states created in $K$-shell photoionization. Finally, the virtual absence of satellite lines in the Auger spectrum awaits further scrutiny. It appears feasible to address these many open questions; their solution will doubtless advance the still rudimentary understanding of open-shell systems and provide base data for pinpointing atomic features in Auger spectrometry of various materials. 
We thank F. Larkins for providing us with the results of his Hartree-Fock calculations prior to publication. We also thank C. A. de Lange for the use of his microwave generator and discharge tube. This work was supported by the National Science Foundation under Grant No.
PHY-8907286 and by the U.S. Department of Energy, Basic Energy Sciences, under Contract No. DE-AC0584-OR21400 with Martin Marietta Energy Systems, Inc. The Synchrotron Radiation Center is operated under National Science Foundation Grant No. DMR-8821625.
[1] T. Åberg and G. Howat, in Corpuscles and Radiation in Matter I, Handbuch der Physik Vol 31, edited by W. Mehlhorn (Springer-Verlag, Heidelberg, 1982), pp. 469-619.

[2] W. Mehlhorn, in Atomic Inner-Shell Physics, edited by B. Crasemann (Plenum, New York, 1985), Chap. 4.

[3] M. O. Krause and C. D. Caldwell, Phys. Rev. Lett. 59, 2376 (1987).

[4] M. Rødbro, R. Bruch, and P. Bisgaard, J. Phys. B 12, 2413 (1979).

[5] N. Stolterfoht, Phys. Rep. 146, 317 (1987).

[6] C. P. Bhalla, J. Electron Spectrosc. Relat. Phenom. 7, 287 (1975).

[7] M.-H. Chen, F. P. Larkins, and B. Crasemann, At. Data Nucl. Data Tables 45, 1 (1990).

[8] M. O. Krause, T. A. Carlson, and A. Fahlman, Phys. Rev. A 30, 1316 (1984).

[9] P. vd Meulen, M. O. Krause, and C. A. de Lange, Phys. Rev. A 43, 5997 (1991).

[10] K. Pearson, Biometrika 16, 157 (1924).
[11] C. E. Moore, Atomic Energy Levels, National Bureau of Standards (U.S.) Circ. No. 467 (U.S. GPO, Washington, DC, 1976), Vol. 1.

[12] F. P. Larkins (private communication).

[13] M. Larsson, P. Baltzer, S. Svensson, B. Wannberg, N. Mårtensson, A. Naves de Brito, N. Correia, M. P. Keane, M. Carlsson-Göthe, and L. Karlsson, J. Phys. B 23, 1175 (1990).

[14] W. E. Moddeman, T. A. Carlson, M. O. Krause, B. P. Pullen, W. E. Bull, and G. K. Schweitzer, J. Chem. Phys. 55, 2317 (1971).

[15] F. Wuilleumier and M. O. Krause, J. Electron Spectrosc. Relat. Pheonom. 15, 15 (1979); Phys. Rev. A 10, 242 (1974).

[16] M. O. Krause, T. A. Carlson, and W. E. Moddeman, J. Phys. (Paris) Colloq. 32, C4-139 (1971).

[17] C. D. Caldwell, M. G. Flemming, M. O. Krause, P. vd Meulen, C. Pan, and A. F. Starace, Phys. Rev. A 41, 542 (1990). 